\section{DETECTION OF SUSPECTED CHILD ABUSE AT EMERGENCY DEPARTMENTS, A MULTI- CENTER STUDY}

E. Louwers ${ }^{1}$, I. Korfage ${ }^{1}$, M. Affourtit ${ }^{2}$, H. Moll ${ }^{2}$, H. De Koning ${ }^{1}$

${ }^{1}$ Public Health, Erasmus MC, University Medical Center Rotterdam, ${ }^{2}$ Paediatrics, Erasmus

MC Sophia Childrens Hospital, Rotterdam, Netherlands Antilles

Background: Recognizing child abuse in an early stage or preventing recurrent abuse is crucial but difficult. Implementing screening guidelines for child abuse at emergency departments (ED) could be effective to increase the detection rate. We assessed detection rates of child abuse at ED of seven hospitals with and without screening guidelines in the Netherlands.

Methods: During six months data on demographics, diagnosis and suspected child abuse were collected for all children up to 18 years old visiting the ED. Beforehand 'having clear screening guidelines' was operationalized as a completion rate of a checklist for alarming factors for child abuse in at least $10 \%$ of the ED visits.

Results: 24,472 children visited the ED of whom $54 \%$ visited an ED with screening guidelines for child abuse. In 52 children $(0.2 \%)$ child abuse was suspected at the ED, in $40(77 \%)$ of these cases a checklist for alarming factors was completed versus $19 \%$ of completed cases in the total sample. Children in whom a suspicion of abuse arose at the ED were younger and more often male than the general ED population. The majority concerned physical abuse. In hospitals with screening guidelines for child abuse the detection rate was higher $(0.3 \%)$ compared to hospitals without a screening policy $(0.1 \%, p<0.001)$.

Conclusions: In a timeframe of six months a suspicion of child abuse arose at the ED in $0.2 \%$ of all visiting children. Numbers of detected abuse were very low, but improvements are likely if uniform screening guidelines are present.

\section{FEVER OF UNKNOWN ORIGIN IN INFANTS YOUNGER THAN 12 WEEKS}

V. Van Gorp ${ }^{1}$, G. van Berlaer ${ }^{2}$, S. Hachimi-Idrissi ${ }^{1}$, I. Hubloue ${ }^{2}$

${ }^{1}$ Paediatric Critical Care Department, ${ }^{2}$ Department of Emergency and Disaster Medicine, Universitair Ziekenhuis Brussel, Brussels, Belgium

Background and aims: In young infants presenting to the emergency department (ED) with fever it is mostly very difficult to determine the origin of the febrile illness, and many are empirically treated with antibiotics before diagnosis is clear. Fear of developing sepsis and/or meningitis force paediatricians to complete a workup, before starting empirical antibiotics. It is still undetermined which investigations are helpful or mandatory.

Methods: We analysed characteristics, initial symptoms, extent of workup, final diagnoses and treatment of all infants presenting with fever of unknown origin (FUO) to the ED over three years.

Results: Out of 43696 paediatric patients, 252 were younger than 12 weeks and presented with FUO. Less than half received complete investigational workup.

Most reliable information was gathered from the physical exam: clinical signs as dyspnea, cyanosis or palor, impaired peripheral circulation and irritability were most associated with critical illness. CRP-elevation was one of the most consistent parameters of serious illness.

In $65 \%$ cultures remained negative. Over $80 \%$ was admitted, of which $57 \%$ empirically treated with broad-spectrum-antibiotics. Only $34 \%$ were initially observed, and solely treated after clinical deterioration or culture positivation. Most frequent diagnosis was URTI, followed by viral infection, 15\% were discharged without diagnosis. Median length of stay was 4.5 days (range $0-14$ days). None of the infants deceased.

Conclusions: More children than necessary are empirically treated, but if antibiotic treatment is postponed, close observation and monitoring are mandatory. Any clinical deterioration should lead to treatment, as physical signs are most reliable to suspect serious illness. 